Rev. Int. Contam. Ambie. 37, 273-279, 2021

https://doi.org/10.20937/RICA.53745

\title{
REGISTRO PRELIMINAR DE MICROPLÁSTICOS EN FECAS DEL LEÓN MARINO SUDAMERICANO (Otaria byronia [de Blainville 1820]) RECOLECTADAS EN PUNTA SAN JUAN, PERÚ
}

Preliminary record of microplastics in scats of south American sea lion (Otaria byronia [de Blainville 1820]) collected in Punta San Juan, Peru

\author{
Félix AYALA*, Marco CARDEÑA y Susana CÁRDENAS-ALAYZA
}

Centro para la Sostenibilidad Ambiental, Universidad Peruana Cayetano Heredia. Av. Armendáriz 445, Lima 15074, Perú.

* Autor para correspondencia: viridisechura@gmail.com

(Recibido: octubre de 2019; aceptado: agosto de 2020)

Palabras clave: desechos marinos, pinnípedos, reserva marina, contaminación marina

\section{RESUMEN}

Los residuos plásticos han sido identificados en excretas de otáridos alrededor del mundo. No obstante, en el Perú los reportes previos de estos contaminantes en Otaria byronia no han sido evaluados. En el presente trabajo se reporta la presencia y forma de microplásticos (MP) en las heces de esta especie, recolectadas dentro de un área natural protegida al sur del Perú. Se logró identificar un total de 47 partículas plásticas clasificadas como fibras y fragmentos, cuya media longitudinal fue de 3.02 y $1.19 \mathrm{~mm}$, respectivamente. La procedencia de estas partículas plásticas milimétricas en las fecas de $O$. byronia se desconoce; sin embargo, otros estudios han asociado la incidencia de MP en la dieta de los animales. Se sugiere mejorar las técnicas de detección de MP en futuros estudios para continuar los esfuerzos de investigación de contaminantes en animales silvestres marinos.

Key words: marine debris, pinnipeds, marine reserve, marine pollution

\begin{abstract}
Plastic waste has been identified in otariid excreta around the world. However, in Peru, previous reports of these contaminants in O. byronia have not been evaluated. In this study, we report the presence and shapes of microplastics (MPs) found in scat samples of this species, collected within a natural protected area in southern Peru. A total of 47 plastic particles classified as fibers and fragments were identified, whose average length was 3.02 and 1.19 , respectively. The origin of these millimetric plastic particles in O. byronia scats is unknown; however, other studies have associated the incidence of MPs to the diet of the animals. For future studies, we suggest improving the detection techniques of MPs to continue research efforts on contaminants in free ranging aquatic species.
\end{abstract}




\section{INTRODUCCIÓN}

Los microplásticos (MP) afectan a una gran diversidad de organismos marinos (Guzzetti et al. 2018). Estas partículas de plástico de tamaños inferiores a $5 \mathrm{~mm}$ de diámetro (GESAMP 2015, Boucher y Friot 2017) pueden estar ampliamente biodisponibles en los distintos niveles tróficos (Wright 2013, Law y Thompson 2014). Su tendencia a seguir disgregándose por factores asociados a la fotodegradación (Browne et al. 2007), así como su acción mecánica (Cooper y Corcoran 2010) y por otros organismos biológicos (Dawson et al. 2018), actualmente los han convertido en residuos omnipresentes (Peng et al. 2020). Se originan además por agregados en productos cosméticos, exfoliantes faciales y productos de cuidado personal (Napper et al. 2015, Guerranti et al. 2019), cuyo uso y mala praxis generan su ingreso a posterior a las aguas residuales que terminan desembocado en los océanos (van Wezel et al. 2016). La presencia de estos residuos se ha constatado en fauna marina, por ejemplo en crustáceos (Devriese et al. 2015, Jamieson et al. 2019), peces (Ory et al. 2018, Herrera et al. 2019), aves (Herzke et al. 2015, Rossi et al 2019) y mamíferos (Eriksson y Burton 2003, Nelms et al. 2019).

De los residuos sólidos, el plástico ha sido uno de los materiales que mayor afectación ha ocasionado a la fauna marina (Rochman et al. 2016). Recientemente, Kühn y van Franeker (2020) señalaron que más de 900 especies de animales marinos han sufrido ya sea enredamiento por materiales plásticos o la ingesta de éstos, siendo las aves, tortugas y mamíferos los grupos faunísticos más perjudicados.

La mayor parte de los estudios que relacionan la interacción de mamíferos marinos con residuos marinos presentan descripciones completas tanto del origen del material (Denuncio et al. 2011, de Stephanis et al. 2013, Besseling et al. 2015, Lusher et al. 2018) como de las potenciales vías de incorporación de estos materiales al océano y a los organismos, ya sea a través de aguas residuales, la escorrentía de ciudades (Moore 2008, Cesa et al. 2017) o la transferencia trófica (Eriksson y Burton 2003, Nelms et al. 2018). Sin embargo, son pocos los estudios del impacto del plástico en pinnípedos (Butterworth 2016, Nelms et al. 2019) en vida libre.

En una de las primeras evaluaciones sobre microplásticos en muestras fecales de lobos marinos (Arctocephalus tropicalis y A.gazella) se examinaron 145 fecas y se hallaron 164 partículas con longitud media de $4.1 \mathrm{~mm}$ (Eriksson y Burton 2003). Por otro lado, Denuncio et al. (2017) examinaron el contenido estomacal en 133 lobos marinos sudamericanos (Arctocephalus australis) hallados muertos en playas al norte de Argentina y sur de Brasil, de los cuales el $7 \%$ presentaron desechos marinos plásticos. Asimismo, existen estudios realizados en cautiverio con focas grises (Halichoerus grypus) en los cuales se demostró de forma empírica la transferencia trófica de partículas plásticas desde los peces hasta los depredadores superiores, encontrándose una correlación entre el tipo de polímero en las presas y en las fecas de los animales (Nelms et al. 2018).

Por lo anterior, el presente estudio se realizó a manera de piloto con la finalidad de responder algunas interrogantes como: ¿existen contaminantes plásticos milimétricos detectables en las heces del león marino sudamericano? Si es así, ¿cuáles son las formas de MP más frecuentes en las muestras? Dado que el estudio de muestras fecales puede emplearse como un método de monitoreo de la exposición a MP en pinnípedos (Nelms et al. 2018, Donohue et al. 2019, Pérez-Venegas et al. 2020), este estudio examina las heces de uno de los depredadores tope en uno de los sitios más productivos del mundo (Bakun y Weeks 2008).

\section{MATERIALES Y MÉTODOS}

Las muestras fecales de $O$. byronia fueron recolectadas en la playa N7 de Punta San Juan (PSJ) (15 22'00" S-75 12'00" O) (Fig. 1), una península que forma parte de la Reserva Nacional Sistema de Islas, Islotes y Puntas Guaneras (RNSIIPG). Dentro de PSJ, la playa N7 es la playa reproductiva con la mayor congregación de individuos de $O$. byronia, y es utilizada exclusivamente por esta especie.

Se recolectaron 10 muestras de fecas el martes 28 de febrero de 2017, como parte de un estudio piloto del Programa Punta San Juan, autorizado en la Resolución Jefatural No. 019-2016-SERNANP-RNSIIPG. Se procuró recoger excretas recién evacuadas por el león marino sudamericano.

Las muestras fueron recolectadas usando una espátula metálica (evitando siempre el uso de herramientas de plástico) y envueltas herméticamente en papel aluminio; posteriormente se preservaron en una bolsa Ziploc etiquetada individualmente. Ese mismo día fueron colocadas en un recipiente de vidrio con agua para disolver la materia orgánica durante aproximadamente $7 \mathrm{~h}$. Tres unidades fecales se dejaron individualmente en bandejas de acero inoxidable y se examinaron al tercer día de haber sido colocadas en agua potable. 


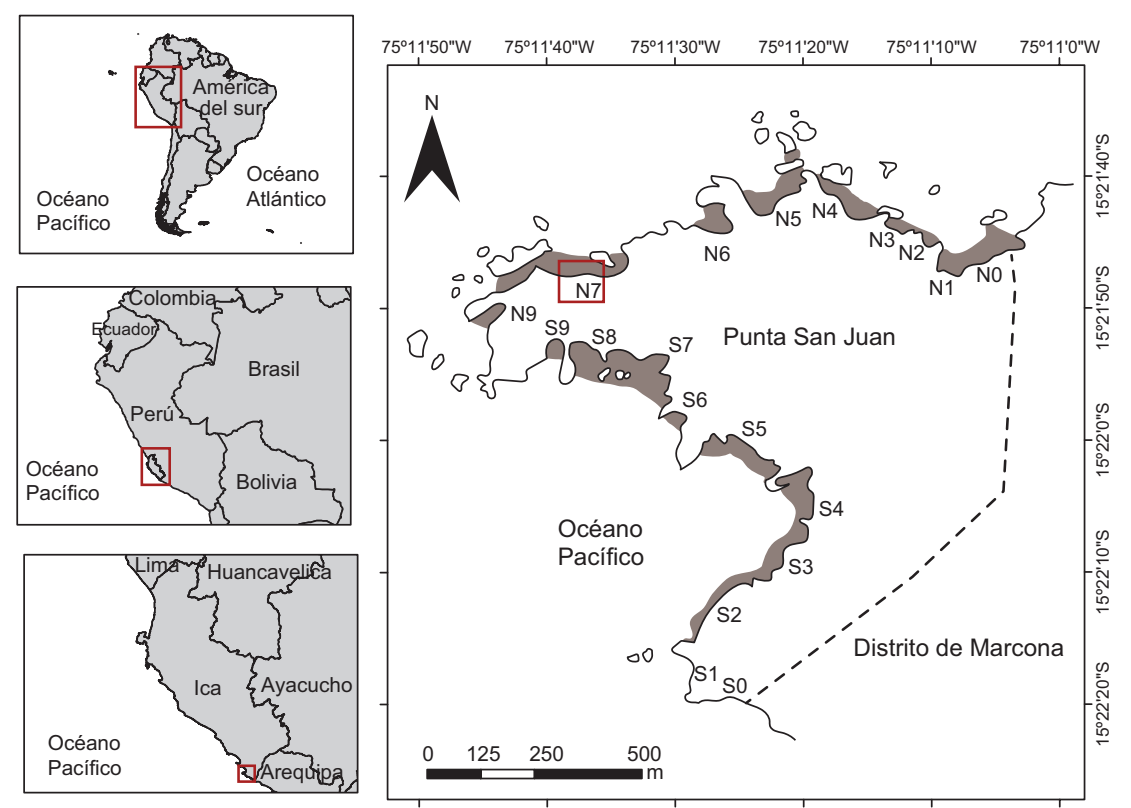

Fig. 1. Ubicación de la playa de estudio N7 en Punta San Juan, Departamento de Ica, Perú.

Se terminó con el proceso de limpieza y segregación de partes duras en la totalidad de las muestras en 12 días. Para la segregación de las partes duras, cada feca fue lavada con ayuda de un tamiz metálico de tres niveles con especificaciones ASTM E-11.

Luego de ser tamizados, los residuos sólidos milimétricos fueron transportados a placas Petri y examinados con un estereoscopio $(3 \times)$ para la respectiva clasificación por forma de los MP. Se tomaron fotografías de los fragmentos (formas irregulares) y de las fibras con el software ToupView; después se analizaron con el programa ImageJ utilizando una hoja milimetrada como referencia de escala para medir estos residuos plásticos. Finalmente, se almacenaron en viales de vidrio.

\section{RESULTADOS}

De las muestras fecales de $O$. byronia analizadas, el $80 \%(n=8)$ presentó MP, encontrándose un total de 47 partículas plásticas con tamaños inferiores a $5 \mathrm{~mm}$. De éstas, el $91 \%(\mathrm{n}=43)$ fueron fragmentos plásticos y el $9 \%(n=4)$ fibras. La media longitudinal fue de $1.19 \mathrm{~mm}(\mathrm{SD} \pm 0.68)$ y $3.02 \mathrm{~mm}(\mathrm{SD} \pm 1.12)$ para los fragmentos y fibras, respectivamente. Los fragmentos plásticos se encontraron en el $80 \%$ de las muestras fecales, en tanto que las fibras plásticas se observaron en el $30 \%$ de las muestras. El color predominante fue el azul con variaciones en las tonalidades (Fig. 2). También se encontraron restos blancos, transparentes y marrones; pero no fueron considerados en la contabilización final de los resultados dado que se considera que existe una posible fuente de error en la identificación y diferenciación de los mismos dentro de la materia orgánica.

En el total de las muestras tamizadas se identificaron 21 otolitos de anchoveta (Engraulis ringens), seis mandíbulas de cefalópodos (especie sin identificar), así como 37 cristalinos de peces y abundantes restos de munida (Pleuroncodes monodon).

\section{DISCUSIÓN}

Este trabajo reporta el primer registro de MP hallados a partir de muestras fecales de $O$. byronia en el Perú y a su vez dentro de un área natural protegida. En las muestras de heces del león marino sudamericano los MP de coloración azul fueron las más predominantes y también los de formas más irregulares. Un trabajo anterior centrado en la isla Macquarie (Australia) examinó en detalle la presencia de estos fragmentos en las fecas de lobos marinos (A. tropicalis y A. gazella), encontrando que las pequeñas piezas de plástico presentaban una forma irregular con coloraciones de colores blanco, marrón, azul, verde y amarillo (Eriksson y Burton 2003); sin embargo, en las muestras de heces de los otáridos analizadas en PSJ, los resultados coinciden en parte 


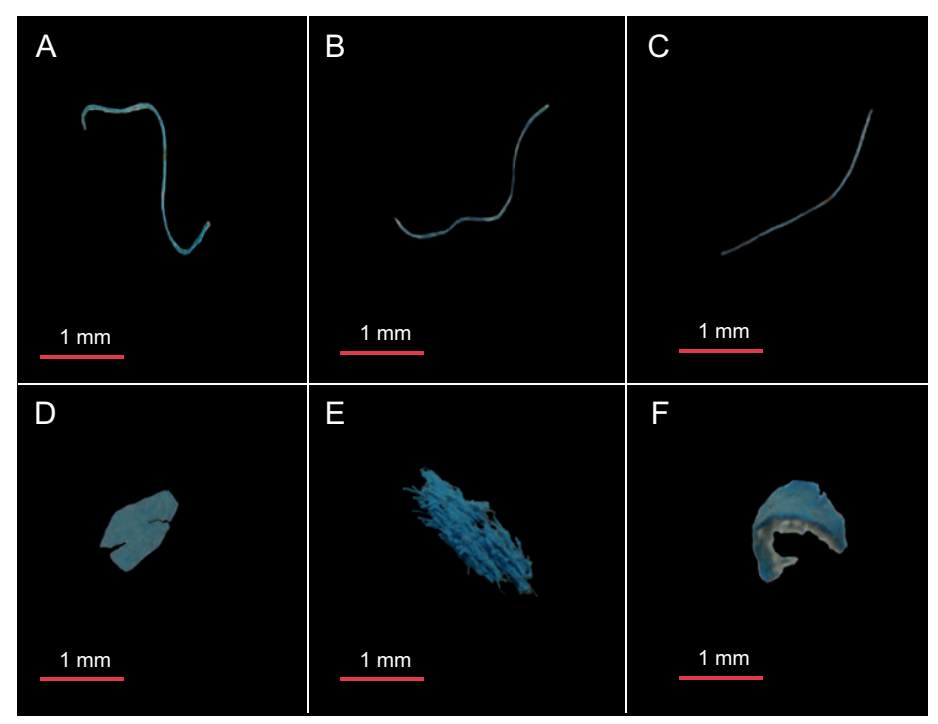

Fig. 2. (a-c) fibras plásticas, (d-f) fragmentos de plástico identificados en las muestras de Otaria byronia.

con investigaciones más recientes que han detectado mayormente MP azules en distintas especies marinas, tales como crustáceos (Devriese et al. 2015, Jamieson et al. 2019), peces (Ory et al. 2017, Herrera et al. 2019), focas y cetáceos (Nelms et al. 2019), al igual que en fecas de otras especies de lobos marinos (Pérez-Venegas et al. 2018, 2020), en sedimentos y en agua de mar (Gago et al. 2018). Dado el carácter piloto del presente estudio, existen limitaciones en la metodología y por ende existe una fuente de error en la identificación correcta de MP de otros colores, pudiéndose haber subestimado el número total de MP en las muestras analizadas. Además, los MP de coloración roja, por ejemplo, se pueden confundir fácilmente con los restos de munida ( $P$. monodon), crustáceo de coloración rojiza presente en la dieta de O. byronia.

Por otra parte, es necesario tomar mejores medidas que eviten la contaminación cruzada de muestras; por ejemplo, se sabe que el agua corriente de consumo humano puede ser otra potencial fuente de MP (Koelmans et al. 2019, Shruti et al. 2020), y que el aire puede ser un generador de contaminación por partículas en suspensión (Gasperi et al. 2018). Para ello se sugiere el uso de muestras control que evalúen los posibles contaminantes en agua $u$ otras potenciales fuentes de contaminación a lo largo del proceso que puedan influir en los resultados finales (Donohue et al. 2019). Si bien en el presente estudio los MP hallados no parecían ser de materiales textiles (como los de la ropa), no es posible descartar totalmente algún tipo de contaminación aérea. A su vez, se debe tener en cuenta el uso de agua en las muestras de futuros trabajos.

La mayoría de trabajos publicados hasta el momento sobre transferencia trófica de microplásticos se han desarrollado en laboratorio y en especies de bajo nivel trófico, tanto para especies de sistemas dulceacuícolas como marinas (e.g., cangrejos y peces), y se ha demostrado que este tipo de organismos pueden verse afectados negativamente por la transferencia trófica de partículas plásticas a través de los eslabones alimenticios, sufriendo efectos tales como cambios bioquímicos, de comportamiento e histológicos (Farrell y Nelson 2013, Chae et al. 2018, Nelms et al. 2018). Sin embargo, se desconocen los efectos crónicos potenciales de los MP en organismos tróficos superiores como mamíferos marinos (Nelms et al. 2019).

En las muestras de heces de león marino sudamericano de PSJ se registraron otolitos, cristalinos y mandíbulas de cefalópodos. Estas presas pertenecen a grupos taxonómicos (peces y calamares) que basan su alimentación en señales visuales (Shaw y Day 1994) y pueden estar consumiendo residuos plásticos con el alimento. Asimismo, Araya (1983) reportó la presencia de plásticos en el contenido estomacal de calamares voladores (Ommastrephes bartrami), lo cual sustenta la presencia de MP en taxones similares a los que forman parte de las presas habituales de $O$. byronia. También hay estudios que reportan la ingesta de MP (especialmente fibras) de color rojo por parte de peces al confundirlas con su alimento (Mizraji et al. 2017). De igual modo, existe evidencia de que el 
cangrejo rojo (Pleuroncodes planipes), congénere de la munida ( $P$. monodon), puede presentar MP en sus contenidos gastrointestinales (Choy et al. 2019).

Diversos estudios publicados sobre otáridos en América del Sur han encontrado desechos plásticos en contenidos estomacales y en heces de las especies A. australis y A. tropicalis. Estos contaminantes se encontraron en la categoría de micro, meso y macroplásticos e incluían redes, filamentos, fibras y bolsas plásticas (Oliveira et al. 2008, Denuncio et al. 2017, Pérez-Venegas et al. 2018). Asimismo, Pérez-Venegas et al. (2020) encontraron plásticos micrométricos en muestras de heces en cuatro especies/subespecies de otáridos que incluían muestras procedentes de león marino sudamericano recolectadas en Chile. El trabajo consideró la posibilidad de la contaminación de MP por transferencia trófica. Por el contrario, no se han publicado registros de MP en esta especie en Perú, por lo que consideramos que este trabajo contribuye a reducir el actual vacío de información sobre este tema.

Finalmente, consideramos necesario continuar con este tipo de estudios para entender con qué frecuencia inciden los MP en la dieta de O. byronia. Recomendamos implementar técnicas de filtración al vacío con compuestos químicos como hidróxido de potasio $(\mathrm{KOH})$ y peróxido de hidrogeno $\left(\mathrm{H}_{2} \mathrm{O}_{2}\right)$ - empleados recientemente en degradación de muestras fecales de lobos marinos (Pérez-Venegas et al. 2018, Donohue et al. 2019) —, con el fin de mejorar el aislamiento de MP en las muestras y acceder a plásticos de menor tamaño, posibilitando así la clasificación de MP por origen y forma (Germanov et al. 2018, Pérez-Venegas et al. 2020). Con relación a la identificación del tipo de polímero encontrado en las excretas, es necesario el uso de técnicas analíticas como la espectrofotometría transformada de Fourier, ya probada y validada en muestras de pinnípedos (e.g., Eriksson y Burton 2003, Nelms et al. 2018, Pérez-Venegas et al. 2020). Por último, es necesario ampliar el número muestral de fecas recolectadas y, de ser posible, hacerlo de manera sistemática para evaluar la variación temporal de la incidencia de MP en esta especie a lo largo del año y en sus principales especies presa.

\section{AGRADECIMIENTOS}

Los trabajos de monitoreo e investigación realizados por el Programa Punta San Juan del Centro para la Sostenibilidad Ambiental fueron posibilitados por convenios de colaboración interinstitucional entre la Universidad Peruana Cayetano Heredia y el SERNANP y Agrorural. Agradecemos a ambas instituciones su colaboración. Asimiso, agradecemos al consorcio de zoológicos que apoyan las operaciones del Programa Punta San Juan (Saint Louis Zoo, Chicago Zoological Society, Kansas City Zoo y Woodland Park Zoo). Su apoyo permitió la estadía de los especialistas en campo y la logística asociada a la presente investigación.

\section{REFERENCIAS}

Araya H. (1983). Fishery, biology, and stock assessment of Ommastrephes bartrami in the North Pacific Ocean. Mem. Natl Mus. Victoria 44, 269-283. https://doi. org/10.24199/j.mmv.1983.44.22

Bakun A. y Weeks S.J. (2008). The marine ecosystem off Peru: What are the secrets of its fishery productivity and what might its future hold? Prog. Oceanogr. 79 (2-4), 290-299. https://doi.org/10.1016/j.pocean.2008.10.027

Besseling E., Foekema E.M., van Franeker J.A., Leopold M.F., Kühn S., Rebolledo E.L.B., Heße E., Mielke L., Ijzer J., Kamminga P. y Koelmans A.A. (2015). Microplastic in a macro filter feeder: Humpback whale Megaptera novaeangliae. Mar. Pollut. Bullet. 95 (1), 248-252. https://doi.org/10.1016/j.marpolbul.2015.04.007

Boucher J. y Friot D. (2017). Primary microplastics in the oceans: A global evaluation of sources. Gland, Switzerland, $46 \mathrm{pp}$.

Browne M.A., Galloway T. y Thompson R. (2007). Microplastic - an emerging contaminant of potential concern? Integr. Environ. Assess. Manag. 3 (4), 559561. https://doi.org/10.1002/ieam.5630030412

Butterworth A. (2016). A review of the welfare impact on pinnipeds of plastic marine debris. Front. Mar. Sci. 3 (149), 1-10. https://doi.org/10.3389/fmars.2016.00149

Cesa F.S., Turra A. y Baruque-Ramos J. (2017). Synthetic fibers as microplastics in the marine environment: A review from textile perspective with a focus on domestic washings. Sci. Total Environ. 598, 1116-1129. https:// doi.org/10.1016/j.scitotenv.2017.04.172

Chae Y., Kim D., Kim S.W. y An Y.J. (2018). Trophic transfer and individual impact of nano-sized polystyrene in a four-species freshwater food chain. Sci. Rep. 8 (284), 1-11. https://doi.org/10.1038/s41598-017-18849-y

Choy C.A., Robison B.H., Gagne T.O., Erwin B., Firl E., Halden R.U., Hamilton J.A., Katija K., Lisin S.E., Rolsky C. y van Houtan K.S. (2019). The vertical distribution and biological transport of marine microplastics across the epipelagic and mesopelagic water column. Sci. Rep. 9 (7843), 1-9. https://doi. org/10.1038/s41598-019-44117-2 
Cooper D.A. y Corcoran P.L. (2010). Effects of mechanical and chemical processes on the degradation of plastic beach debris on the island of Kauai, Hawaii. Mar. Pollut. Bull. 60 (5), 650-654. https://doi.org/10.1016/j. marpolbul.2009.12.026

Dawson A.L., Kawaguchi S., King C.K., Townsend K.A., King R., Huston W.M. y Bengtson N.S.M. (2018). Turning microplastics into nanoplastics through digestive fragmentation by Antarctic krill. Nat. Commun. 9 (1001), 1-8. https://doi.org/10.1038/s41467-018-03465-9

De Stephanis R., Giménez J., Carpinelli E., GutiérrezExpósito C. y Cañadas A. (2013). As main meal for sperm whales: Plastics debris. Mar. Pollut. Bullet. 69 (1-2), 206-214. https://doi.org/10.1016/j.marpolbul.2013.01.033

Denuncio P., Bastida R., Dassis M., Giardino G., Gerpe M. y Rodríguez D. (2011). Plastic ingestion in Franciscana dolphins, Pontoporia blainvillei (Gervais and d'Orbigny, 1844), from Argentina. Mar. Pollut. Bullet. 62 (8), 1836-1841. https://doi.org/10.1016/j. marpolbul.2011.05.003

Denuncio P., Mandiola M.A., Salles S.B.P., Machado R., Ott P.H., Oliveira L.R. y Rodríguez D. (2017). Marine debris ingestion by the South American Fur Seal from the Southwest Atlantic Ocean. Mar. Pollut. Bull. 122 (1-2), 420-425. https://doi.org/10.1016/j. marpolbul.2017.07.013

Devriese L.I., van der Meulen M.D., Maes T., Bekaert K., Paul-Pont I., Frère L., Robbens J. y Vethaak A.D. (2015). Microplastic contamination in brown shrimp (Crangon crangon, Linnaeus 1758) from coastal waters of the Southern North Sea and Channel area. Mar. Pollut. Bull. 98 (1-2), 179-187. https://doi. org/10.1016/j.marpolbul.2015.06.051

Donohue M.J., Masura J., Gelatt T., Ream R., Baker J.D., Faulhaber K. y Lerner D.T. (2019). Evaluating exposure of northern fur seals, Callorhinus ursinus, to microplastic pollution through fecal analysis. Mar. Pollut. Bull. 138, 213-221. https://doi.org/10.1016/j. marpolbul.2018.11.036

Eriksson C. y Burton H. (2003). Origins and biological accumulation of small plastic particles in fur seals from Macquarie island. Ambio 32 (6), 380-384. https://doi. org/10.1579/0044-7447-32.6.380

Farrell P. y Nelson K. (2013). Trophic level transfer of microplastic: Mytilus edulis (L.) to Carcinus maenas (L.). Environ. Pollut. 177, 1-3. https://doi.org/10.1016/j. envpol.2013.01.046

Gago J., Carretero O., Filgueiras A.V. y Viñas L. (2018). Synthetic microfibers in the marine environment: A review on their occurrence in seawater and sediments. Mar. Pollut. Bull. 127, 365-376. https://doi. org/10.1016/j.marpolbul.2017.11.070
Gasperi J., Wright S.L., Dris R., Collard F., Mandin C., Guerrouache M., Langlois V., Kelly F.J. y Tassin B. (2018). Microplastics in air: Are we breathing it in? Curr. Opin. Environ. Sci. Health 1, 1-5. https://doi. org/10.1016/j.coesh.2017.10.002

Germanov E.S., Marshall A.D., Bejder L. Fossi M.C. y Loneragan N.R. (2018). Microplastics: No small problem for filter-feeding megafauna. Trends Ecol. Evol. 33 (4), 227-232. https://doi.org/10.1016/j. tree.2018.01.005

GESAMP (2015). Sources, fate and effects of microplastics in the marine environment: A global assessment. Joint Group of Experts on the Scientific Aspects of Marine Environmental Protection (IMO/FAO/UNESCOIOC/UNIDO/WMO/IAEA/UN/UNEP/UNDP. Reports and Studies. London, UK, 98 pp.

Guerranti C., Martellini T., Perra G., Scopetani C. y Cincinelli A. (2019). Microplastics in cosmetics: Environmental issues and needs for global bands. Environ. Toxicol. Pharmacol. 68, 75-79. https://doi. org/10.1016/j.etap.2019.03.007

Guzzetti E., Sureda A., Tejada S. y Faggio C. (2018). Microplastic in marine organism: Environmental and toxicological effects. Environ. Toxicol. Pharmacol. 64, 164-171. https://doi.org/10.1016/j. etap.2018.10.009

Herrera A., Ŝtindlová A., Martínez I. Rapp J., RomeroKutzner V., Samper, M.D., Montoto T., AguiarGonzález B., Packard T. y Gómez M. (2019). Microplastic ingestion by Atlantic chub mackerel (Scomber colias) in the Canary Islands coast. Mar. Pollut. Bull. 139, 127-135. https://doi.org/10.1016/j. marpolbul.2018.12.022

Herzke D., Anker-Nilssen T., Nøst T.H., Götsch A., Christensen-Dalsgaard S., Langset M., Fangel K. y Koelmans A.A. (2015). Negligible impact of ingested microplastics on tissue concentrations of persistent organic pollutants in northern fulmars off coastal Norway. Environ. Sci. Technol. 50 (4), 1924-1933. https://doi.org/10.1021/acs.est.5b04663

Jamieson A.J., Brooks L.S.R., Reid W.D.K., Piertney S.B., Narayanaswamy B.E. y Linley T.D. (2019). Microplastics and synthetic particles ingested by deep-sea amphipods in six of the deepest marine ecosystems on Earth. Royal Soc. Open Sci. 6 (2), 1-11. https://doi. org/10.1098/rsos. 180667

Kühn S. y van Franeker. (2020). Quantitative overview of marine debris ingested by marine megafauna. Mar. Pollut. Bull. 151, 110858. https://doi.org/10.1016/j. marpolbul.2019.110858

Koelmans A.A., Nor N.H.M., Hermsen E., Kooi M., Mintenig S.M. y de France J. (2019). Microplastics in freshwaters and drinking water: Critical review and 
assessment of data quality. Water Res. 155, 410-422. https://doi.org/10.1016/j.watres.2019.02.054

Law K.L. y Thompson R.C. (2014). Microplastics in the seas. Science 345 (6193), 144-145. https://doi. org/10.1126/science. 1254065

Lusher A.L., Hernández-Milian G., Berrow S., Rogan E. y O'Connor I. (2018). Incidence of marine debris in cetaceans stranded and bycaught in Ireland: Recent findings and a review of historical knowledge. Environ. Pollut. 232, 467-476. https://doi.org/10.1016/j.envpol.2017.09.070

Mizraji R., Ahrendt C., Pérez-Venegas D., Vargas J., Pulgar J., Aldana M., Ojeda F.P., Duarte C. y Galbán-Malagón C. (2017). Is the feeding type related with the content of microplastics in intertidal fish gut? Mar. Pollut. Bull. 116 (1-2), 498-500. https://doi.org/10.1016/j. marpolbul.2017.01.008

Moore C.J. (2008). Synthetic polymers in the marine environment: A rapidly increasing, long-term threat. Environ. Res. 108 (2), 131-139. https://doi.org/10.1016/j. envres.2008.07.025

Napper I.E., Bakir A., Rowland S.J. y Thompson R.C. (2015). Characterisation, quantity and sorptive properties of microplastics extracted from cosmetics. Mar. Pollut. Bull. 99 (1-2), 178-185. https://doi. org/10.1016/j.marpolbul.2015.07.029

Nelms S.E., Galloway T.S., Godley B.J., Jarvis D.S. y Lindeque P.K. (2018). Investigating microplastics trophic transfer in marine top predators. Environ. Pollut. 238, 999-1007. https://doi.org/10.1016/j.envpol.2018.02.016

Nelms S.E., Barnett J., Brownlow A., Davison N.J., Deaville R., Galloway T.S. Lindeque P.K., Santillo D. y Godley B.J. (2019). Microplastics in marine mammals stranded around the British coast: Ubiquitous but transitory? Sci. Rep. 9 (1075), 1-8. https://doi. org/10.1038/s41598-018-37428-3

Oliveira L.D., Ott P.H. y Malabarba L.R. (2008). Ecologia alimentar dos pinípedes do Sul do Brasil e uma avaliação de suas interações com atividades pesqueiras. En: Ecologia de mamíferos (dos Reis N.R., Ed.). Technical Books, Londrina, Brasil, pp. 97-116.

Ory N.C., Sobral P., Ferreira J.L. y Thiel M. (2017). Amberstripe scad Decapterus muroadsi (Carangidae) fish ingest blue microplastics resembling their copepod prey along the coast of Rapa Nui (Easter Island) in the South Pacific subtropical gyre. Sci. Total Environ. 586, 430-437. https://doi.org/10.1016/j. scitotenv.2017.01.175

Ory N., Chagnon C., Felix F., Fernández C., Ferreira J.L., Gallardo C., Ordóñez O.G., Henostroza A., Laaz E., Mizraji R., Mojica H., Haro V.M., Medina L.O., Preciado M., Sobral P., Urbina M.A. y Thiel M. (2018). Low prevalence of microplastic contamination in planktivorous fish species from the southeast Pacific Ocean. Mar. Pollut. Bull. 127, 211-216. DOI: https:// doi.org/10.1016/j.marpolbul.2017.12.016

Peng L., Fu D., Qi H., Lan C.Q., Yu H. y Ge C. (2020). Micro- and nano-plastic in marine environment: Source, distribution and threats - A review. Sci. Total Environ. 698, 134254. https://doi.org/10.1016/j.scitotenv.2019.134254

Pérez-Venegas D.J., Seguel M., Pavés H. Pulgar J., Urbina M., Ahrendt C. y Galbán-Malagón C. (2018). First detection of plastic microfibers in a wild population of South American fur seals (Arctocephalus australis) in the Chilean Northern Patagonia. Mar. Pollut. Bull. 136, 50-54. https://doi.org/10.1016/j.marpolbul.2018.08.065

Pérez-Venegas D.J., Toro-Valdivieso C., Ayala F., Brito B., Iturra L., Arriagada M., Seguel M., Barrios C., Sepúlveda M., Oliva D., Cárdenas-Alayza S., Urbina M.A., Jorquera A., Castro-Nallar E. y Galbán-Malagón C. (2020). Monitoring the occurrence of microplastic ingestion in Otariids along the Peruvian and Chilean coasts. Mar. Pollut. Bull. 153, 110966. https://doi. org/10.1016/j.marpolbul.2020.110966

Rochman C.M., Browne M.A., Underwood A.J., van Franeker J.A., Thompson R.C. y Amaral-Zettler L.A. (2016). The ecological impacts of marine debris: Unraveling the demonstrated evidence from what is perceived. Ecology 97 (2), 302-312. https://doi. org/10.1890/14-2070.1

Rossi L.C., Scherer A.L. y Petry M.V. (2019). First record of debris ingestion by the shorebird American (Haematopus palliates) on the Southern coast of Brazil. Mar. Pollut. Bull. 138, 235-240. https://doi.org/10.1016/j. marpolbul.2018.11.051

Shaw D.G. y Day R.H. (1994). Colour- and form-dependent loss of plastic micro-debris from the North Pacific Ocean. Mar. Pollut. Bull. 28 (1), 39-43. https://doi. org/10.1016/0025-326x(94)90184-8

Shruti V.C., Pérez-Guevara F. y Kutralam-Muniasamy G. (2020). Metro station free drinking water fountain - A potential "microplastics hotspot" for human consumption. Environ. Pollut. 261, 114227. https://doi. org/10.1016/j.envpol.2020.114227

Van Wezel A., Caris I. y Kools S.A.E. (2016). Release of primary microplastics from consumer products to wastewater in the Netherlands. Environ. Toxicol. Chem. 35 (7), 1627-1631. https://doi.org/10.1002/ etc. 3316

Wright S.L., Thompson R.C. y Galloway T.S. (2013). The physical impacts of microplastics on marine organisms: A review. Environ. Pollut. 178, 483-492. https://doi. org/10.1016/j.envpol.2013.02.031 